\title{
Turbulent Mixing of two-layer Stratified Fluid
}

\author{
J. A. Whitehead \\ Department of Physical Oceanography, MS\#21 \\ Woods Hole Oceanographic Institution \\ Wood Hole MA 02543 \\ Email: jwhitehead@whoi.edu \\ Ian Stevenson \\ Interdepartmental Neuroscience Program \\ Northwestern University \\ Searle Building 5-474 \\ 320 East Chicago Ave. \\ Chicago, IL 60611 - 3010 \\ 9 November, 2007
}

\begin{abstract}
A two-layer salt-stratified tank of water was mixed by turbulence generated by many excursions of a horizontally moving vertical rod. The objective is to observe the timedependent response of the mean density field for ranges of Richardson number $\mathrm{Ri}>0.9$ and Reynolds Number Re $>600$. As the density profile of the fluid gradually evolves from a single step to a mixed state over a wide range of time, there is almost perfect collapse of all the profiles to one universal profile as a function of a similarity variable. Although the turbulent diffusion is not constant, the value in the limit of small stratification has similar magnitude to values found by others.
\end{abstract}




\section{Introduction}

Strong currents spontaneously develop near topography in the ocean ${ }^{1,2}$ and lead to mixing. This led to our renewed interest in mixing within layered fluid. Numerous experiments have been performed using turbulence in salt water, air or other gases ${ }^{3,4}$ with either discrete layers or continuous stratification. The turbulence is generated in tunnels by instability of shear flow, or by flowing through grids, and in tanks by moving rakes, grids or rods.

A primary governing parameter is the Reynolds number $\operatorname{Re}=U d / v$ for the turbulence, which needs to be high enough for turbulence to exist (roughly $>500$ ). A second governing parameter is either a Richardson number $R i=g \Delta \rho d / \rho_{0} U^{2}$ for layered fluid, or an internal Richardson number $R i i=N^{2} d^{2} / U^{2}$ for smoothly stratified fluid. In these formulas, the acceleration of gravity is $g, \Delta \rho$ is the initial density difference if there are two layers, $\rho$ is density of the fluid, $\rho_{0}$ is its average, $d$ is the length scale of the turbulence source, $U$ is the speed of the turbulence source, $v$ is the viscosity of the fluid, and $N^{2}=-\left(g / \rho_{0}\right) d \rho / d z$ is stratification frequency. The Prandtl number and Schmidt number are also relevant. They express the ratio of viscosity to diffusivity of the property that causes density change, (temperature or concentration, respectively). They are secondary in importance for high Reynolds number, and low Richardson number. Effects of their variation are not covered here. We restrict attention here to turbulence generated in a tank. Experiments with a local source of turbulence inside one or both layers produce particularly good data for two layers if $R i>1$. Data are accurate because the interface remains sharp and each layer remains well mixed. Buoyancy flux (gravity times the rate of density flux), which is easily estimated by measuring the evolution of the density field, falls off as $R i$ increases. For $R i<1$, the results have more scatter and require longer averaging time because the mixed layers are filled with fine 
structure, the interface is greatly distorted, and the change in density with time is rapid compared to eddy overturning time, unless the tank is very deep.

Experiments beginning with uniform stratification and with active mixing at all depths of the fluid give good measurements for all ranges of $R i i$. For $R i i<1$, mixed boundary layers form at the bottom and top, and expand downward and upward. Meanwhile, in the interior there is a gradual decrease of $N$ (rather than an increase as is the case for localized mixing). For $R i i>1$ the density field evolves differently, with a spontaneous growth of overlying mixed layers separated by "staircase" jumps in density ${ }^{5-8}$, similar to models ${ }^{9-12}$ that predict instability of turbulent uniformly stratified fluid. For smaller Rii and $\mathrm{Re}<800$, streamwise vortices and boundary mechanisms produce layers.

The present experiment combines the initial condition of two homogeneous layers with stirring throughout the entire fluid depth. Previous experiments used a grid dropped once through two layers with differing salinity ${ }^{13}$. Section 2 describes an experiment that generates many such stirring events instead of only one. The evolving density profile collapses to one profile (Section 4) through the use of a similarity variable (Section 3).

\section{The experiment}

A $0.6 \mathrm{~m}$ high acrylic tank (Fig. 1) with a horizontal cross-section $0.1 \mathrm{~m} \times 0.2 \mathrm{~m}$ was initially filled to a $0.25 \mathrm{~m}$ depth of salt water $\left(\mathrm{NaCl}\right.$, density $\rho$ varied from $1030 \mathrm{mg} / \mathrm{m}^{3}$ to 1200 $\mathrm{kg} / \mathrm{m}^{3}$ in 11 different runs). Then, an equal thickness of fresh water was carefully added using a float-mechanism to create two distinct layers of differing density with a sharp interface between them. Temperature effects were negligible because both layers were within $1.0{ }^{0} \mathrm{C}$ of room temperature. A conductivity microprobe measured density profiles with resolution to $0.001 \mathrm{~m}$. 
A vertical mixing rod extending throughout the depth of the tank generated the turbulence. It traversed back and forth along a $0.15 \mathrm{~m}$ long track in the wide direction of the tank. After recording the initial density profile, mixing was started and after a certain number of rod excursions back and forth, the mixing was stopped. A profile was taken after a 2-minute settling period. Episodes of mixing followed by a profile continued. The cumulative number of rod excursions during any part of a run is $n$. A complete experiment had up to $n_{\max }=300$ rod excursions and up to 80 profiles.

We take rod diameter $d$ to be the length scale for turbulence. Three different diameters were used $\left(1.27,1.8\right.$, and $\left.2.68 \times 10^{-2} \mathrm{~m}\right)$. The range of the values of $U$ was $0.05-0.071 \mathrm{~m} / \mathrm{s}$. Using the values for reference density and viscosity at $20{ }^{0} \mathrm{C}$, the 11 experimental runs had a Reynolds number range of $638<\mathrm{Re}<1765$. The Richardson number range of the initial conditions for all the runs is $0.93<R i<4.84$.

At the beginning of a run, the local values of Rii are very large in the vicinity of the interface and virtually zero in each layer. After mixing starts, the interface widens (Fig. 2), the maximum value of $R i i$ at the interface decreases for each excursion, and Rii is nonzero throughout the fluid. An average value of Rii is Riia $\cong d R i / D$, where $D$ is water depth $(0.50$ $\mathrm{m})$. The range for these experiments is $0.024<$ Riia $<0.174$. This suggests that the experiment lies outside the range $R i i>1$ where layer formation is found ${ }^{6,7}$.

Density values of the endpoints are correct within $0.003 \%$. The microprobe yields calibrated density for each profile that is precise within one part in $10^{4}$ of the endpoints. To correct for a small probe drift, we centered the profiles by adding a small uniform shift ${ }^{6,7,10}$. The centered profiles were averaged over 5 points taken at $0.001 \mathrm{~m}$ intervals to smooth noise. Because of instrumental noise of the same magnitude, the scatter of the differences between 
closely spaced points or sequential profiles needed to determine gradients or buoyancy flux was considerable.

\section{A simple model}

The conservation of density obeys

$$
\frac{\partial \rho}{\partial t}=-\frac{\partial F_{d}}{\partial z}
$$

with $F_{d}$ the density flux in the $z$ direction, and $z$ positive upward. Two models of flux will be discussed. One simple model of the flux is proportional to density gradient and is $F_{d}=-c U d \partial \rho / \partial z$. This is unique as long as the length of the rod is ignored, because in that case $U d$ is the only combination of rod properties with the proper dimensions. The proportionality constant $c$ is determined by the turbulent mixing. Starting with two layers of differing density, this has a complementary error function solution ${ }^{11}$

$$
\rho=\rho_{0}+\frac{\Delta \rho z}{2|z|} \operatorname{cerf}\left(\frac{|z|}{2 \sqrt{c U d t}}\right)
$$

We also consider a second flux model with the increase of flux for small stratification and decrease for large stratification. Since it is only a model, we set the equivalent of $c$ equal to one.

$$
F_{d}=-U d \frac{\frac{\partial \rho}{\partial z}}{1+\left(\frac{g}{\rho_{0}} \frac{\partial \rho}{\partial z}\right)^{2} \frac{d^{4}}{U^{4}}}
$$

This expression with a vertical derivative of equation (3.1) reduces to

$$
\frac{\partial N^{2}}{\partial t}=U d \frac{\partial^{2}}{\partial z^{2}} \frac{N^{2}}{1+\frac{N^{4} d^{4}}{U^{4}}}
$$


The equation is nondimensionalized using the time scale $\frac{d}{U}$ and vertical length scale $d$ to produce an equation for $\operatorname{Rii}(z, t)$

$$
\frac{\partial R i i}{\partial t^{\prime}}=\frac{\partial^{2}}{\partial z^{\prime 2}} \frac{R i i}{1+R i i^{2}}
$$

which can be converted to an ordinary differential equation using the similarity variable ${ }^{11}$

$$
\begin{aligned}
& \eta=\frac{z^{\prime}}{2 \sqrt{t^{\prime}}},(\text { used to derive }(3.2)) . \text { The similarity equation is } \\
& -\eta \frac{d}{d \eta} R i i=\frac{1}{2} \frac{d^{2}}{d \eta^{2}} \frac{R i i}{1+R i i^{2}} .
\end{aligned}
$$

Note that the similarity transformation could be done for any flux that is a function of only Rii. For the evolution of a dense fluid below a lighter fluid separated by a sharp interface, the initial condition is $\operatorname{Rii}(z, t=0)=\delta(z)$ where $\delta$ is the Dirac delta function. We don't know of any analytical solutions for (3.5) or (3.6), but they are easily solved numerically. To begin the numerical integration, the numerical delta function was a smooth function having a finite width of ten dimensionless depth units with a peak value of $R i i=1$. If $R i i$ is given a greater peak value, vertical buoyancy flux becomes suppressed at the interface, leading to the possibility of either instability ${ }^{8,9}$ or a shock. This limit is not investigated because the laboratory experiment showed no signs of layers or a shock. The similarity approach is valid everywhere within an unbounded region. However, it works well within restricted regions for a finite time. To show this, an integration of (3.5) is shown in Fig. 3a. After a short adjustment period of about $t^{\prime}=5$, the profiles collapse to a universal similarity profile (Fig. 3b) until $t^{\prime}=200$, after which the density change reached the top and bottom ends of the numerical region and the collapse to the similarity variable became degraded. 


\section{Application of the similarity scaling to data}

In the experiment, time is taken to be linearly proportional to the number of rod excursions $n$. Shadowgraphs show that turbulence is still present over the entire cycle so this approximation seems to be quite good. All of the experiments showed strikingly good data collapse using the similarity variable of depth divided by $n^{1 / 2}$. Fig. 4 shows all profiles for three runs. To the left is the physical depth and to the right is the depth divided by $n^{1 / 2}$. In looking at individual profiles, it was found that the first few profiles don't overlap well, just as in the numerical model. To clearly show the closest overlap, Fig. 5 shows selected profiles for two cases: the top example corresponds to panels a and b in Fig. 4. The other examples shown in Fig. 4 had equally good collapse. The bottom panels correspond to an additional run. For the selected profiles, the first profile was made a few traverses after the start of the experiment, the cluster of profiles is from midway through the experiment, and one profile is from near the end. The fit is only slightly bigger than the scatter in the data and therefore almost perfect. Also, the fit is almost as good as the fit of different times in the numerical example shown in Figure $3 b$. Thus, the collapse to the similarity variable is as good as would be expected even if there were perfect agreement with the scaling theory.

Figure 6 shows a comparison of theory and data by overlaying the similarity complementary error function, the numerical similarity curve from Fig. 3b, and the collapsed data from Fig. 5b. A time-step for the experiment is one traverse multiplied by the time scale $d / U$ and then multiplied by some unknown constant. Therefore, the vertical scale of the collapsed data from the experiment is not precisely known. Because of this, the vertical axis values are not specified and the depth scales for the two theoretical curves were stretched to have 
the same slope as the experimental data at mid-depth. The error function and the numerical similarity curve are virtually identical. Both theories do not overlap the experimental data.

Using the similarity variable, the thickness of the transition region between the upper and lower layer in Figs. 5b,d can be used to infer a value of eddy diffusivity. In dimensional units, the similarity variable is $\eta=z / 2 \sqrt{c d U t}$ so that $c U d$ serves as a substitute for the diffusivity. Figure 6 shows that the region with $90 \%$ of the change between the upper and lower layer is found within about $\eta= \pm 0.05$. Let us compare this to the complementary error function equation (3.2). In that case, we can define the transition layer to lie within about $\eta= \pm 1$. For our present experiment to have the same dimensionless layer thickness, we require that $c=0.05^{2}$ and the similarity variable is $\eta=z / 2 \sqrt{0.05^{2} d U t}$ so the effective diffusivity is about $2.5 \times 10^{-3} U d$. This is smaller than the value $\kappa_{\text {eff }}=6.7 \times 10^{-3} U_{0} M$ found by Holford and Linden ${ }^{8}$ with grid stirring, where $M$ is the grid spacing and $U_{0}$ is peak grid speed. Since there is no direct geometrical correspondence between the rod used in this experiment and their grids, these two results agree as well as can be expected.

Buoyancy flux and potential energy evolution were also calculated from the data. Since the probe was unable to traverse the entire depth of the tank, an estimate was required for flux into the bottom of the test area from the change in density in deeper depths. The flux and energy evolution for the 11 experiments using these estimates were inconsistent with each other and are not satisfactory enough to be included. However, the fluxes in the measured region $F_{u}$ for various times during each individual run exhibited close overlap. One case is shown in Fig. 7. The flux $F_{u}$ is determined by integrating (3.1) upward and ahead in time for one data set starting with $F_{u}=0$ at the bottom of the measured region $(z=0)$, then multiplying by $g$ and dividing by 
initial density difference. Profiles of $F_{u}$ (Fig. 7a) and profiles of smoothed stratification parameter Rii (Fig. 7b) can be combined to show flux versus stratification (Fig. 7c). There is a monotonic increase of $F_{u}$ at small $R i i$, and a leveling out of flux (with no decrease) for Rii $>0.05$. Therefore, the results are consistent with the shape given by equation (3.3), but except to the left of the maximum flux in equations (3.3-3.6). This is the regime for the numerical integration described in section 3. The values of flux are smaller than those in other laboratory experiments. Clearly this is due in part to absence of flux traveling upward from the unmeasured region below the test area in this experiment. The lack of fine structure in these experiments and the numerical model is thus consistent with the regime diagram of Holford and Linden $^{8}$, who report no layers in that range of Rii. Finally, estimates of the amount of turbulent kinetic energy going to mixing processes show that a maximum of approximately $5 \%$ of the kinetic energy generated by the moving rod increases potential energy.

\section{Conclusions}

Using similarity theory in a model equation for the evolution of a stratified density distribution agrees with experimental results. The collapse of data to a similarity variable is almost perfect from $n=8$ to $n=300$. Therefore, the experimental results are consistent with the concept that such a flux law is a function of Rii alone. This similarity-based approach, as verified by laboratory experiments, supports the use of a similarity variable for tracer mixing in

oceanography ${ }^{15}$. Although the experiment obtained reasonable results, quantitative precision of flux laws was not obtained because the density was not sampled at all levels. 


\section{Acknowledgements}

Ian Stevenson was supported as a summer student fellow of Woods Hole Oceanographic Institution. The laboratory experiment was supported by the Ocean and Climate Change Institute of the Woods Hole Oceanographic Institution.

\section{References}

1. J. A. Whitehead, "Internal hydraulic control in rotating fluids- applications to oceans," Geophysical and Astrophysical Fluid Dynamics 48, 169-192 (1989).

2. A. M. Thurnherr, L. C. St. Laurent, K. G. Speer, J. M. Toole, and J. R. Ledwell, "Mixing associated with sills in a canyon on the midocean ridge flank," J. Phys. Oc. 35, 1370$1381(2005)$.

3. J. S. Turner, "Buoyancy Effects in Fluids," Cambridge University Press, 368 pp. (1973).

4. H. J. S. Fernando, “Mixing in Stratified Fluids,” Ann. Rev. Fluid Mech. 23, 455-493 (1991).

5. S. A. Thorpe, "On the layers produced by rapidly oscillating a vertical grid in a uniformly stratified fluid,” J. Fluid Mech. 124, 391-409 (1982).

6. B. R. Ruddick, T. J. McDougall and J. S. Turner, "The formation of layers in a uniformly stirred density gradient,” Deep-Sea Res. 356, 597-609 (1989).

7. Y-G. Park, J. A. Whitehead, and A. Gnanadesikan, 1994. "Turbulent mixing in stratified fluids: layer formation and energetics,” J. Fluid Mech. 279, 279-311 (1994).

8. J. M. Holford, and P. F. Linden, “Turbulent mixing in a stratified fluid,” Dyn. Atmos. Oceans 30, 173-198 (1999).

9. O. M Phillips, “Turbulence in a strongly stratified fluid - is it unstable?” Deep-Sea Res. 19, 79-81 (1972). 
10. E. S. Posmentier, "The generation of salinity fine structure by vertical diffusion," J. Phys. Oceanogr. 7, 298-300 (1977).

11. G. I Barenblatt, M. Bertsch, R. Dalpasso, V. M. Prostokishin, and M. Ughi, "A mathematical model of turbulent heat and mass transfer in stably stratified shear flow," J. Fluid Mech. 253, 341-358 (1993).

12. N. J. Balmforth S. G. L. Smith and W. R. Young, "Dynamics of interfaces and layers in a stratified turbulent fluid” J. Fluid Mech. 355, 329-358 (1998).

13. P. F. Linden, "Mixing across a density interface produced by grid turbulence," J. Fluid Mech. 100, 691 (1980).

14. D. Turcotte and G. Schubert, “Geodynamics: Applications of Continuum Physics to Geological Problems,” Wiley and Sons (1982).

15. J. R. Ledwell, A. J. Watson and C. S. Law, "Mixing of a tracer in the pycnocline," J. Geophys. Res. (O) 103 (C10) 21499-21529 (1998).

\section{Figure Captions}

Figure 1. A diagram of the apparatus.

Figure 2. Density profiles for one run.

Figure 3. (a) Theoretical density profiles for the times $t^{\prime}=5,10,20,40,80,120$ and 200. (b) The profiles plotted against the similarity variable.

Figure 4. Experimental density profiles (left column, a, c, e) and collapsed curves when a similarity variable is used (right column, $b, \mathrm{~d}, \mathrm{f})$. Reynolds and Richardson Numbers are: $(\mathrm{a}, \mathrm{b})$ $\operatorname{Re}=638, R i=0.93$; (c, d) $\operatorname{Re}=900, R i=0.65$; and (e, f) $\operatorname{Re}=1021, R i=1.46$. 
Figure 5. Selected profiles (left) and their collapse when divided by $n^{1 / 2}$ (right). The traverse numbers for panels a, b are: $n=20$ (thin line), $n=41-84$ (cluster of lines), and $n=300$ (thick line). The bottom panels $\mathrm{c}, \mathrm{d}$ are from a run with $\mathrm{Re}=891$ and $R i=1.69$. Traverse numbers are: $n=14$ (thin line), $n=40-60$ (cluster of lines), and $n=336$ (thick line).

Figure 6. The profiles from the similarity error function (black large dashes) the numerical similarity theory (gray) and the experiment curves in Figure $5 b$.

Figure 7. a) Flux versus depth. Flux units are $\mathrm{cm}^{2} \mathrm{~s}^{-3}$. b) Dimensionless stratification (Rii) versus depth. c) Dimensionless flux versus Rii . 


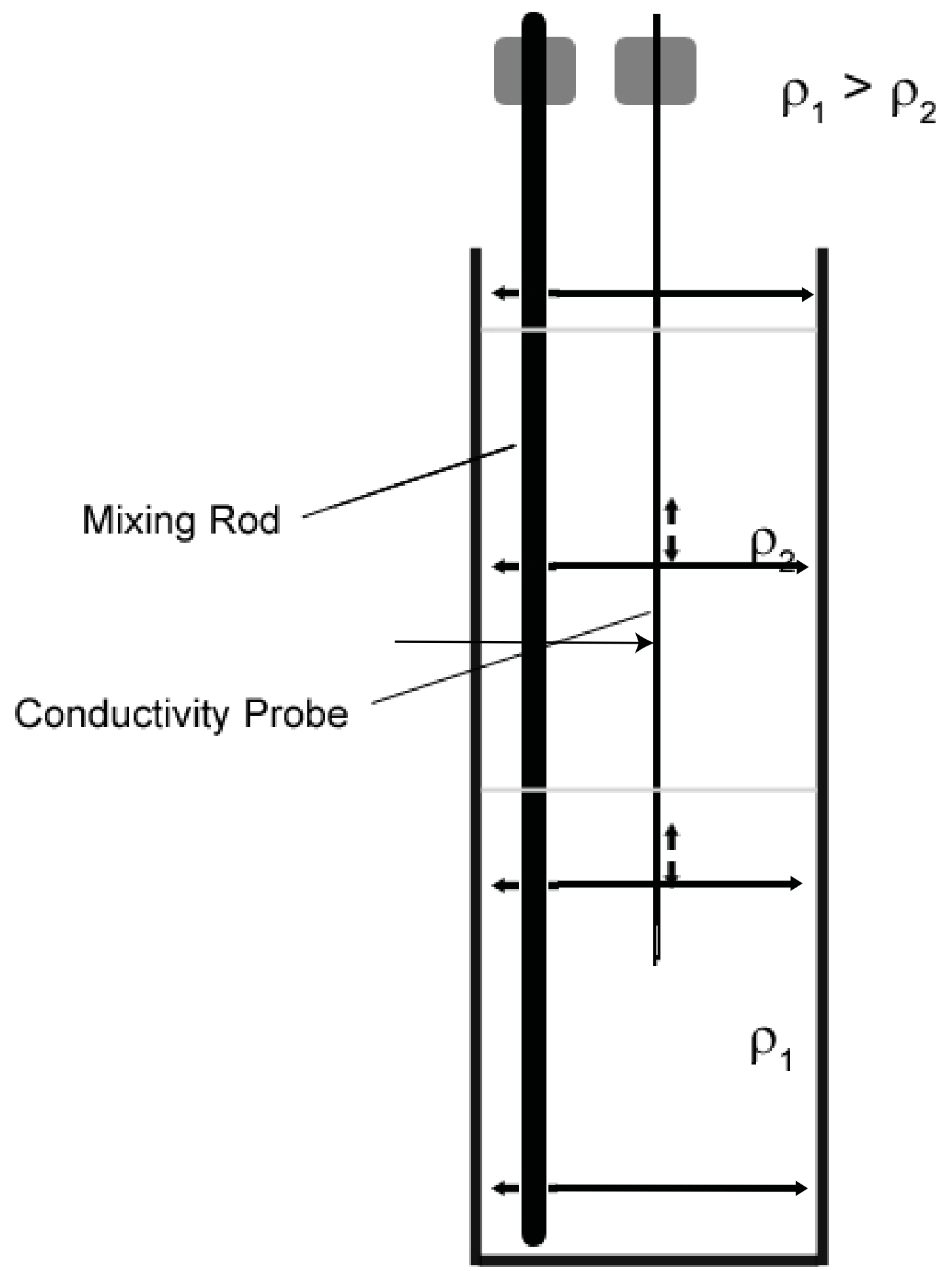




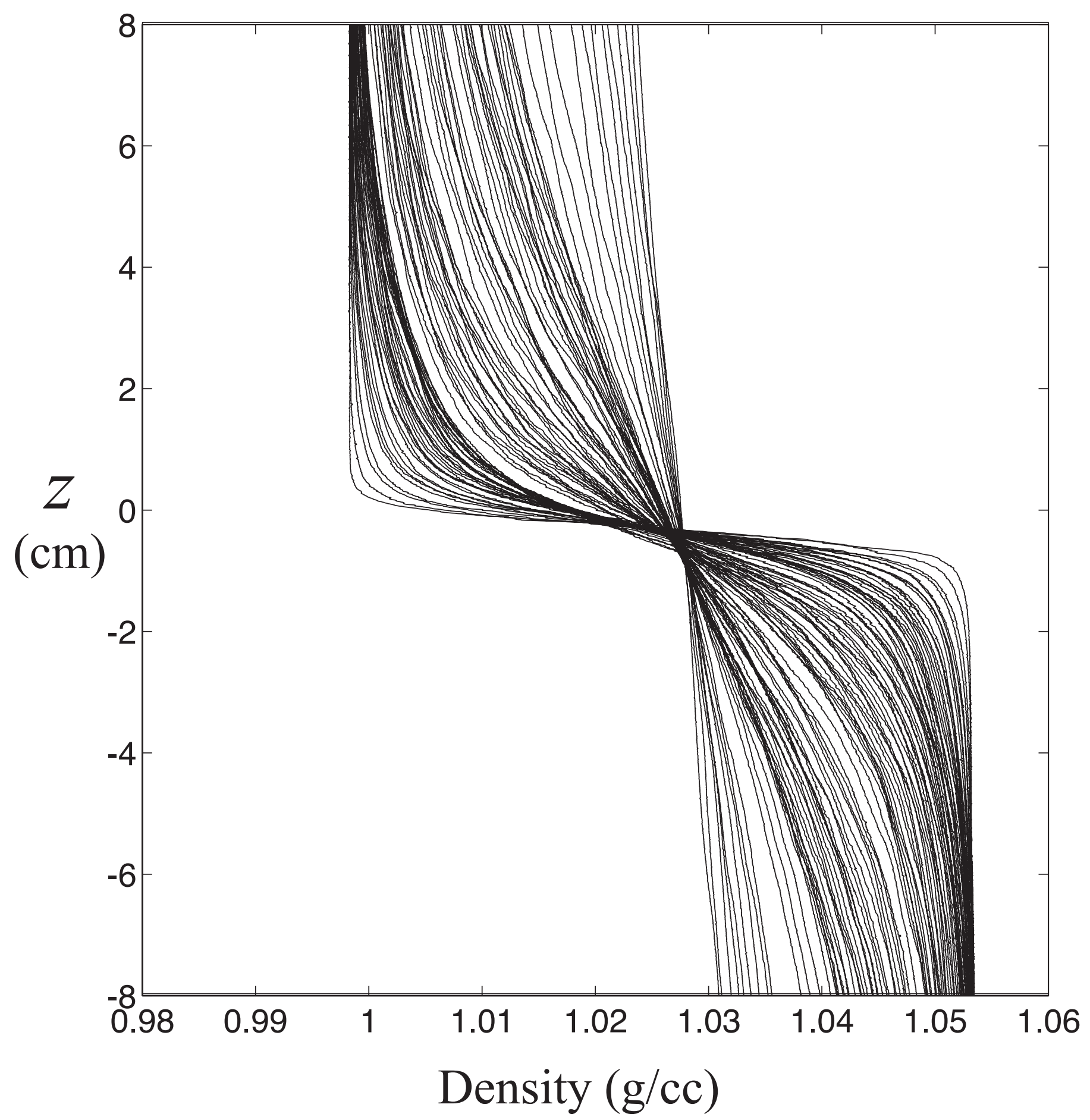



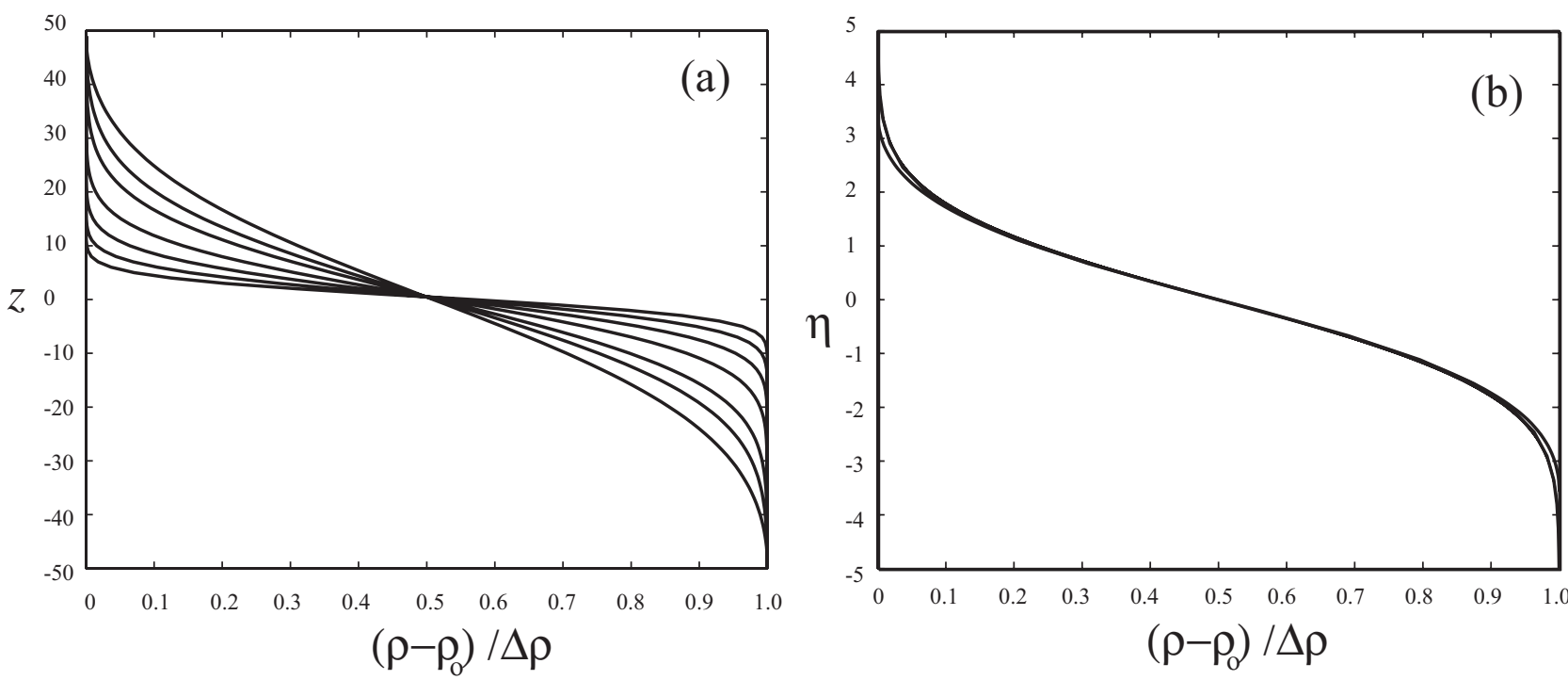


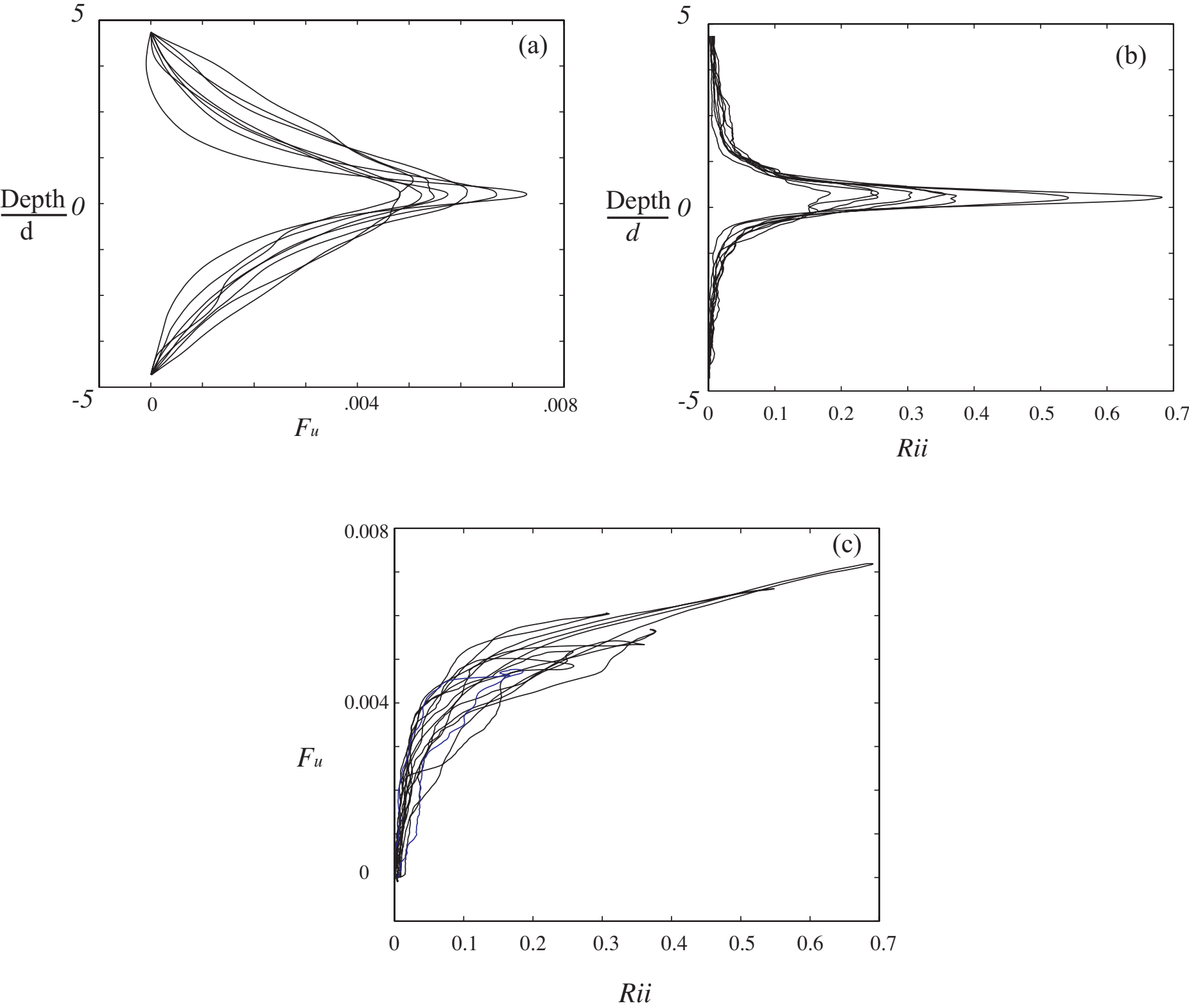